\title{
In vitro cytotoxic, radical scavenging and antimicrobial activities of curcuma mangga valeton and van zijp
}

\begin{abstract}
Curcuma mangga belongs to the family Zingibereae locally known as "mango ginger" or "manggo turmeric". Medicinally, the rhizomes of Curcuma mangga are used for treatment of stomach and chest pains, fever, stomach ulcer and general weakness. It is also used in postpartum treatment, particularly to help in the healing of the womb. The various medicinal purpose of this species inspired further phytochemical studies and potential biological activities. Phytochemical studies reported the isolation of six compounds identifies as curcumin (1), demethoxycurcumin (2), curcumol (3), curdione (4), zederone (5) and $\beta$-Sitosterol (6). Curcumol (3), curdione (4) and zederone (5) are reported to be first isolated from this species. Crude extracts and isolated constituents possessed potential cytotoxic activities against five human cancerous cell lines; promyelocytic leukemia (HL-60), breast cancer (MCF-7), colonic cancer (HT-29), cervical cancer (HeLa) and T-lymphoblastic (CEM-SS). Promising antioxidant activities and remarkable antimicrobial properties are recorded against Methicillin-resistant Staphylococcus aureus (MRSA), Bacillus subtillis and Pseudomonas aeruginosa.
\end{abstract}

Keyword: Curcuma mangga; Cytotoxic; Antioxidant; Antimicrobial; Constituents 